\title{
Getting around cosmic variance
}

\section{Citation}

Kamionkowski, Marc, and Abraham Loeb. 1997. "Getting around Cosmic Variance." Physical Review D 56 (8): 4511-13. https://doi.org/10.1103/physrevd.56.4511.

\section{Permanent link}

http://nrs.harvard.edu/urn-3:HUL.InstRepos:41417255

\section{Terms of Use}

This article was downloaded from Harvard University's DASH repository, and is made available under the terms and conditions applicable to Other Posted Material, as set forth at http:// nrs.harvard.edu/urn-3:HUL.InstRepos:dash.current.terms-of-use\#LAA

\section{Share Your Story}

The Harvard community has made this article openly available.

Please share how this access benefits you. Submit a story.

Accessibility 


\title{
Getting around cosmic variance
}

\author{
Marc Kamionkowski* \\ Department of Physics, Columbia University, 538 West 120th Street, New York, New York 10027 \\ Abraham Loeb \\ Astronomy Department, Harvard University, Cambridge, Massachusetts 02138
}

(Received 16 May 1997)

\begin{abstract}
Cosmic microwave background $(\mathrm{CMB})$ anisotropies probe the primordial density field at the edge of the observable Universe. There is a limiting precision ("cosmic variance") with which anisotropies can determine the amplitude of primordial mass fluctuations. This arises because the surface of last scatter (SLS) probes only a finite two-dimensional slice of the Universe. Probing other SLS's observed from different locations in the Universe would reduce the cosmic variance. In particular, the polarization of CMB photons scattered by the electron gas in a cluster of galaxies provides a measurement of the CMB quadrupole moment seen by the cluster. Therefore, CMB polarization measurements toward many clusters would probe the anisotropy on a variety of SLS's within the observable Universe, and hence reduce the cosmic-variance uncertainty. [S0556-2821(97)04820-0]
\end{abstract}

PACS number(s): 98.70.Vc, 98.90.+s

One of the primary aims of cosmology is recovery of the primordial spectrum of density perturbations which produced the large-scale structure in the Universe today. This spectrum should elucidate whether primordial perturbations were produced by inflation, topological defects, or some alternative mechanism. Although galaxy surveys probe the current mass distribution, the primordial spectrum is best probed by the cosmic microwave background (CMB). Large-angle CMB anisotropies from the Cosmic Background Explorer (COBE) have already probed the spectrum on large distance scales. Furthermore, future experiments such as NASA's Microwave Anisotropy Probe (MAP) [1] and ESA's Planck Surveyor [2] should recover the primordial spectrum to much smaller distance scales with unprecedented precision.

However, there is a fundamental limit to the precision with which the $\mathrm{CMB}$ can recover the amplitude of primordial fluctuations. Theory predicts that the primordial density field was a single realization of some random process. To test the theory, we would want to observe and average over a number of realizations of the random process. However, we have only one Universe to observe, so there will be a sample variance, known as "cosmic variance" in the average we construct.

A given theory provides the three-dimensional power spectrum $P(k)$ as a function of wave number $k$. This specifies roughly the variance in the mass distribution over a comoving-distance scale $\lambda \sim k^{-1}$. Now suppose, for example, that we want to use CMB anisotropies to determine the variance in the mass distribution averaged over spheres of comoving diameter $\lambda$. The CMB probes a spherical surface of last scatter (SLS) at the edge of the observable Universe of comoving radius $R$, as illustrated in Fig. 1. There are only $N \sim 4 \pi(R / \lambda)^{2}$ such volumes probed by our SLS.

\footnotetext{
*Electronic address: kamion@phys.columbia.edu

†Electronic address: aloeb@cfa.harvard.edu
}

Therefore, the fractional precision with which we will be able to determine this variance in the mass distribution is $O\left(N^{-1 / 2}\right)$.

To be more precise, the temperature $T(\hat{\mathbf{n}})$ as a function of direction $\hat{\mathbf{n}}=(\theta, \phi)$ on the sky can be expanded in terms of spherical harmonics

$$
T(\hat{\mathbf{n}})=\sum_{l m} a_{l m} Y_{l m}(\theta, \phi),
$$

with

$$
a_{l m}=\int d \hat{\mathbf{n}} T(\hat{\mathbf{n}}) Y_{l m}^{*}(\hat{\mathbf{n}}) .
$$

These multipole coefficients $a_{l m}$ are distributed with mean $\left\langle a_{l m}\right\rangle=0$ and variance,

$$
\left\langle a_{l^{\prime} m^{\prime}}^{*} a_{l m}\right\rangle=C_{l} \delta_{l l^{\prime}} \delta_{m m^{\prime}},
$$

where the angular brackets denote an average over all realizations of the random field. The set of expectation values $C_{l}$ is the angular power spectrum of the $\mathrm{CMB}$, the projection of the three-dimensional power spectrum $P(k)$ on the twodimensional SLS. Roughly speaking, $C_{l}$ specifies the variance in the mass distribution on a distance scale $\pi R / l$.

To measure a given expectation value $C_{l}$, we would construct the $2 l+1$ (for $m=-l, \ldots, l) a_{l m}$ coefficients from the sky map. The average of the squares of these, $\hat{C}_{l}=\sum_{m=-l}^{l}\left|a_{l m}\right|^{2} /(2 l+1)$ would provide our best estimate for $C_{l}$. However, this average is over a sample with a finite number $(2 l+1)$ of independent terms. Therefore, the precision with which the estimator $\left(\hat{C}_{l}\right)$ will recover the expectation value $\left(C_{l}\right)$ is limited. If the distribution of density perturbations is Gaussian, the $1 \sigma$ cosmic variance with which $C_{l}$ can be estimated is $[2 /(2 l+1)]^{1 / 2} C_{l}$. Although different cosmological models make different predictions for the $C_{l}$ 's measured by COBE (i.e., those for $l \lesssim 15$ ), cosmic variance 


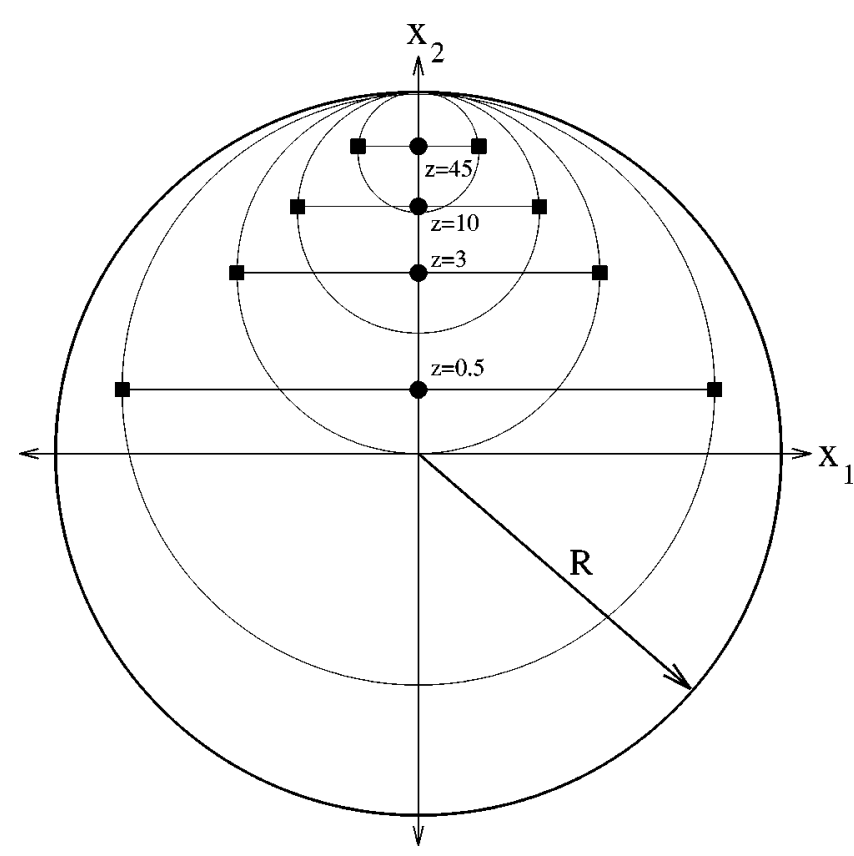

FIG. 1. The $x_{3}=0$ slice of the observable Universe in comoving coordinates. We are at the origin and the $\hat{\mathbf{x}}_{3}$ direction is out of the page. The heavy circle is our surface of last scatter (SLS) located a comoving distance $R$ from our position at the origin. Clusters at redshifts $z=0.5,3,10$, and 45 are located at the comoving positions indicated by the heavy dots along the $+\hat{\mathbf{x}}_{2}$ line of sight (assuming a critical-density universe). The circle centered on each cluster is the SLS seen by it. The filled squares show the primary plane of the quadrupole moment which polarizes the radiation scattered to us.

restricts our ability to discriminate between these different models with COBE measurements.

If we could send observers to numerous distant locations in the Universe and have them report back to us on the CMB anisotropies measured at each of these locations, then we would have additional independent multipole coefficients and therefore be able to overcome the cosmic-variance limit. Although this is not practical, we can probe the anisotropy seen by distant observers.

If one looks at the CMB through a cluster of galaxies, a fraction of the photons have been scattered by the electron gas in the cluster [giving rise to the Sunyaev-Zel'dovich (SZ) effect [3]]. If the radiation incident on the cluster has a quadrupole anisotropy in the plane normal to the line of sight to the cluster, the scattered radiation will be linearly polarized $[4,5]$. Moreover, the polarization vector will be determined by the amplitude and orientation of the quadrupole anisotropy of the incident radiation. By determining the linear polarization of the CMB through a distant cluster, we are measuring two components of the quadrupole moment of the cluster's SLS.

The quadrupole moments we probe in this way are those measured at earlier times when the Hubble radius is smaller than the current Hubble radius. Depending on the cosmology, the expectation value of the quadrupole moment may evolve with time, and it depends on the size of the Hubble radius. Therefore, these quadrupole moments cannot be compared directly with our quadrupole moment, and the cosmicvariance limit to our quadrupole moment cannot be reduced. However, as we discuss below, measurement of many of these quadrupole moments would allow us to map the primordial three-dimensional density field and thereby reduce the cosmic-variance limit to the amplitude of primordial mass fluctuations when smoothed over a given distance scale.

Consider clusters located at the points along the $+\hat{\mathbf{x}}_{2}$ line of sight indicated by the heavy dots in Fig. 1. The sphere centered on each cluster is the SLS observed by it. Clearly, the SLS's of many clusters spread throughout the observable Universe (through many different lines of sight) would span the entire volume of the observable Universe. The lines perpendicular to the line of sight to each cluster indicate the plane of the quadrupole anisotropy probed by the polarization of the scattered radiation we observe. The filled squares show the points on each SLS where those quadrupole moments receive their greatest contribution. Although none of these squares lies closer than $R / \sqrt{2}$ from us (and the closest is for a cluster at $z=3$ ), the volume accessible to the squares is still $\left(1-2^{-3 / 2}\right) \approx 65 \%$ of the observable volume. Although Fig. 1 shows hypothetical clusters with $z>3$, the location of the squares indicates that the accessible volume is equally well probed by a sample of clusters with $z \leqslant 3$. In principle, our approach could be applied to other objects, such as galactic halos which exist at higher redshifts.

Crudely speaking, each polarization signal measures differences in the primordial density at points indicated by the squares in Fig. 1. Therefore, by mapping the polarization of clusters throughout the Universe, we can reconstruct the primordial three-dimensional density field through most of the volume of the observable Universe in much the same way as COBE maps the temperature on the sky from measured temperature differences. Therefore, polarization measurements of the CMB through a number of distant clusters would allow us to probe a larger volume of the observable Universe than that accessible just from our SLS.

The variance in the mass distribution on a comovinglength scale $\lambda$ can be measured with only $N \sim 4 \pi(R / \lambda)^{2}$ independent regions of size $\lambda$ on our SLS. However, the volume of the Universe contains roughly $(R / \lambda)^{3}$ independent regions of size $\lambda$. Therefore, if we can map the primordial density field from cluster polarizations, then the cosmic variance in the determination of the primordial amplitude of density fluctuations could be reduced by up to $O(\sqrt{\lambda / R})$.

In addition to reducing the cosmic variance to the amplitude of primordial inhomogeneities, the measured signals could provide information complementary to that obtained from CMB anisotropy experiments. First, the SLS's for clusters at high redshift will be smaller than ours, so their quadrupole moments probe smaller scales, comparable to those probed by our higher- $l$ moments. Therefore, by observing the redshift dependence of the mean cluster polarization, we obtain an independent measure of the shape of the power spectrum. Furthermore, if the Universe does not have a critical density, additional anisotropies will be produced along the line of sight [6]. Therefore, by comparing the redshift dependence of the cluster polarization signal with the CMB anisotropy measurements, one could separate the line-ofsight contribution from the anisotropy produced at the SLS.

The signal imprinted by reionization on the CMB anisotropies could also probe the density distribution over an extended volume in the Universe. However, the reionization 
signal is integrated over a range of redshifts and cannot provide local information of the type obtained from individual clusters: inference of the three-dimensional density distribution from two-dimensional power spectra requires modeldependent assumptions about the ionization history. It is quite plausible that even before such polarized SZ measurements can be carried out, the three-dimensional mass distribution in the Universe may be mapped over most of the observable Universe with distant galaxies, quasars, x rays, $\mathrm{HI}$, etc. However, these will probe the present (or recent) power spectrum, whereas SZ polarizations will map the primordial mass distribution. Our polarization decomposition of the line-of-sight and SLS anisotropies could independently confirm results from studies of matter/CMB correlations [7].

Finally, we consider the detectability of the signal. The polarization amplitude (in units of the CMB temperature) is expected to be $0.1 \tau \mathrm{Q}$, where $\tau$ is the optical depth of the cluster (as inferred from $\mathrm{x}$-ray observations) and $Q$ is the $\mathrm{CMB}$ quadrupole moment $[4,5]$. Adopting $Q \approx 7 \times 10^{-6}$ and a typical value of $\tau \sim 10^{-2}$ for a rich cluster, we get a polarization signal $\sim 10^{-8}$. Is this detectable? With current technology, no. However, the rate of progress in CMB measurements is phenomenal. A one-year dedicated experiment with a $\mu \mathrm{K} \sqrt{\sec }$ sensitivity could, in principle, measure the above signal for $\sim 10^{3}$ clusters.

The quadrupole signal dominates over competing sources of polarization. The intrinsic CMB polarization fluctuation is practically zero on the $\sim 1^{\prime}$ scale of a cluster core. One could therefore search for the special polarization pattern behind the cluster associated with the scattered quadrupole [5]. A peculiar velocity $v_{\perp}$ of the cluster transverse to the line of sight induces effects of order $0.1\left(v_{\perp} / c\right)^{2} \tau$ or $0.025\left(v_{\perp} / c\right) \tau^{2}$ [8], both of which are much smaller than the quadrupole signal for the characteristic value of $v_{\perp} / c \sim 10^{-3}$. Complementary measurements of radial peculiar velocities, using (the much stronger) kinematic SZ effect, can be combined with the assumption of statistical isotropy to subtract the transverse-velocity contribution to the polarization in a statistical way. The small polarization signals induced by a second scattering of photons from the thermal SZ effect, by scattering of radiation from internal radio sources [8], or by gravitational effects [9], have different frequency or spatial distributions and could be separated from the quadrupole signal.

In conclusion, although cluster polarization is inaccessible with current instruments, its future implementation should give us a way to access other SLS's after the MAP and Planck satellites tell us all there is to learn from ours.

We thank U.-L. Pen and U. Seljak for useful comments. This work was supported at Columbia University by U.S. DOE Contract No. DEFG02-92-ER 40699, NASA ATP Grant No. NAG5-3091, and the Alfred P. Sloan Foundation, and at Harvard University by NASA ATP Grant No. NAG53085 and the Harvard Milton fund.
[1] URL http://map.gsfc.nasa.gov

[2] URL http://astro.estec.esa.nl/SA-general/Projects/Cobras/ cobras.html

[3] R. A. Sunyaev and Ya. B. Zel'dovich, Comments Astrophys. Space Phys. 4, 173 (1972). For a recent review, see, e.g., Y. Rephaeli, Annu. Rev. Astron. Astrophys. 33, 541 (1995).

[4] A. Kosowsky, Ann. Phys. (N.Y.) 246, 49 (1996).

[5] Ya. B. Zel'dovich and R. A. Sunyaev, Sov. Astron. Lett. 6, 285 (1980).
[6] L. A. Kofman and A. A. Starobinsky, Sov. Astron. Lett. 11, 271 (1986); M. Kamionkowski and D. N. Spergel, Astrophys. J. 432, 7 (1994).

[7] R. Crittenden and N. Turok, Phys. Rev. Lett. 76, 575 (1996); M. Kamionkowski, Phys. Rev. D 54, 4169 (1996).

[8] R. Sunyaev and Ya. B. Zel'dovich, Mon. Not. R. Astron. Soc. 190, 413 (1980).

[9] M. Gibilisco, astro-ph/9701203. 